\title{
Silver/Gold Heterometallic Nanostructures and Their Surface Plasmon-Related Behaviors
}

Hyunjoon Song

Department of Chemistry, Korea Advanced Institute of Science and Technology, Daejeon, 305701, Republic of Korea

\begin{abstract}
A combination of silver and gold is demonstrated in nanoscale objects. Silver and gold have similar but distinct chemical and physical properties. We employed three main chemistry related to silver and gold - underpotential deposition, epitaxial growth, and Galvanic replacement. Selective underpotential deposition of silver guided the growth direction of gold and generated gold octahedrons, cuboctahedrons, cubes, and rods. Epitaxial growth of silver on gold decahedral seeds formed silver-gold-silver heterometallic nanorods and wires. Galvanic replacement of silver for gold yielded asymmetric single hollow and symmetric double hollow structures. All silver/gold nanostructures exhibited characteristic optical features in the UV-visNIR ranges. Such structural variation and corresponding optical properties are useful for diverse applications in electronics, photonics, biology, and catalysis.
\end{abstract}

\section{INTRODUCTION}

Heterometallic superlattice structures are particularly of interest because of their multifunctional properties. Since metallic barcodes were synthesized by electrochemical deposition of distinct metal salts inside anodic aluminum oxide templates [1], heterometallic nanorods with various components were fabricated and employed for protein separation, bioassay, and catalytic nanomotors [2]. Compared to the hard template method, synthesis without templates has many advantages including structural variation more than rods, simple process without template dissolution, and large scale production. However, such synthesis has only recently developed, because the reaction condition must be strictly controlled to balance reduction rates of distinct components [3]. In this aspect, silver and gold are excellent components that match together very well. Silver and gold are in Group 11, and have close lattice constants with a small lattice mismatch of $0.2 \%$, which leads to heteroepitaxy on the surface. These two metals also show intense surface plasmon extinctions. There are some differences in chemical reactivity and electrochemical potentials between them, but these make the silver/gold system more exciting. Herein, our recent results related to the coupling of silver and gold components in nanoscale objects are summarized. Gold polyhedrons and rods were synthesized by selective underpotential deposition of silver onto a gold surface in a controlled manner. Silver nanorods and wires were prepared from gold decahedral seeds, which yielded silver-gold-silver heterometallic superlattices through epitaxial growth. Partial Galvanic replacement of gold from silver provided asymmetric and symmetric single and double hollow rods. All these nanostructures showed interesting optical features in the range from visible to near infrared. 


\section{EXPERIMENT}

Control of gold polyhedral shapes by silver underpotential deposition [4]

$\mathrm{A} \mathrm{AgNO}_{3}(99+\%$, Aldrich) solution in 1,5-pentanediol (PD, $0.15 \mathrm{~mL}, 96 \%$, Aldrich) was added to boiling PD $\left(5.0 \mathrm{~mL}\right.$ ). Next, poly(vinyl pyrrolidone) (PVP, $3.0 \mathrm{~mL}, 0.15 \mathrm{M}, \mathrm{M}_{\mathrm{w}}=$ 55,000 , Aldrich) and $\mathrm{HAuCl}_{4}(3.0 \mathrm{~mL}, 0.050 \mathrm{M}, 99.9+\%$, Aldrich) solutions in PD were added periodically every $30 \mathrm{~s}$ over $7.5 \mathrm{~min}$. The resulting mixture was refluxed for $1 \mathrm{~h}$. The final solution was cooled, and the product was purified by a repetitive precipitation/dispersion cycle with ethanol. Different concentrations of $\mathrm{AgNO}_{3}$ solution in PD were used for the synthesis of octahedrons $(1.7 \mathrm{mM}, \mathrm{Ag} / \mathrm{Au}$ molar ratio $=1 / 600)$, cuboctahedrons $(2.5 \mathrm{mM}, \mathrm{Ag} / \mathrm{Au}=1 / 400)$, cubes $(5.0 \mathrm{mM}, \mathrm{Ag} / \mathrm{Au}=1 / 200)$, and higher polygons $(17 \mathrm{mM}, \mathrm{Ag} / \mathrm{Au}=1 / 60)$.

Silver-gold-silver heterometallic nanorods and wires by heteroepitaxial growth $[5,6]$

For nanorods, PVP $(3.0 \mathrm{~mL}, 0.13 \mathrm{M})$ and $\mathrm{AgNO}_{3}(3.0 \mathrm{~mL}, 0.034 \mathrm{M})$ solutions in diethylene glycol (DEG, 99\%, Aldrich) were added to the gold decahedral seed dispersion in DEG $(5.0 \mathrm{~mL})$ periodically every $30 \mathrm{~s}$ over $7.5 \mathrm{~min}$ at boiling temperature. The reaction mixture was refluxed for $1 \mathrm{~h}$. After the reaction, the mixture was cooled and the product was purified by a repetitive dispersion/precipitation cycle with ethanol. For nanowires, PVP $(3.0 \mathrm{~mL}, 0.60 \mathrm{M})$ and $\mathrm{AgNO}_{3}(3.0 \mathrm{~mL}, 0.10 \mathrm{M})$ solutions in ethylene glycol (EG, 99\%, Aldrich) were added to the gold decahedral seed dispersion in EG $(5.0 \mathrm{~mL})$ periodically every $30 \mathrm{~s}$ over $7.5 \mathrm{~min}$, and the reaction mixture was refluxed for $1 \mathrm{~h}$. After the reaction, the mixture was cooled and the product was purified by a repetitive precipitation/dispersion cycle with ethanol.

Asymmetric single and symmetric double hollow formation by Galvanic replacement [7]

An aqueous dispersion of the silver-gold-silver heterometallic nanorods $(1.0 \mathrm{~mL}, 3.8 \mathrm{mM}$ with respect to the silver precursor concentration) was added to the PVP aqueous solution (5.0 $\mathrm{mL}, 20 \mathrm{mM}$ ) at boiling temperature. An appropriate volume of $\mathrm{HAuCl}_{4}$ aqueous solution (6.4 $\mathrm{mM}$ ) was added dropwise to the rod dispersion over $30 \mathrm{~s}$, and the resulting mixture was heated at the reflux condition for additional $3 \mathrm{~min}$. After the reaction, the mixture was cooled and the particles were purified by a repetitive precipitation/dispersion cycle in ethanol. The volumes used for the reactions were $0.040 \mathrm{~mL}$ for the single hollow, and $0.10 \mathrm{~mL}$ for the double hollow structures, respectively.

\section{DISCUSSION}

\section{Control of gold polyhedral shapes by silver underpotential deposition}

Gold polyhedrons were synthesized in PD through a modified polyol process in the presence of PVP [4]. Large gold clumps were generated without the addition of $\mathrm{AgNO}_{3}$ under the present reaction condition. When 1/600 equiv of $\mathrm{AgNO}_{3}$ with respect to the gold precursor concentration was added, the final product was yielded as uniform octahedrons (Figure 1a). The average edge size was estimated to be $100 \pm 10 \mathrm{~nm}$. Increasing the amount of $\mathrm{AgNO}_{3}$ led to edge truncation, and 1/400 equiv addition of the silver precursor afforded cuboctahedrons that covered with cubic and triangular faces (Figure 1b). Fully evolved cubes with an average edge size of $145 \pm 18 \mathrm{~nm}$ were obtained by the addition of 1/200 equiv of the silver precursor (Figure 1c). Above this concentration, a polygonal structure similar to spherical was yielded as monodisperse 
particles with the average diameter of $77 \pm 5 \mathrm{~nm}$. Electron and X-ray diffraction data of the gold polyhedral nanocrystals show that the faces exposed to the polyhedral surface are solely $\{111\}$ for octahedral, $\{111\}$ and $\{100\}$ in an area ratio of 0.58:1 for cuboctahedral, and only $\{100\}$ for cubic nanocrystals, respectively. This structural variation from octahedral to cubic by increasing the silver precursor amount indicates that the silver species generated from $\mathrm{AgNO}_{3}$ are selectively deposited and suppress the surface growth of $\{100\}$. Such silver deposition onto the gold surface exactly matches with a well-known underpotential deposition (UPD) phenomenon in electrochemical processes.

The shape guidance of the silver species on gold nanocrystal growth was also employed for gold nanorod synthesis [8]. 1/200 equiv of $\mathrm{AgNO}_{3}$ with respect to the gold precursor concentration was added to a dispersion of gold decahedral seeds in boiling PD, and PVP and the gold precursor solutions were added to the reaction mixture, followed by heating at reflux temperature. When small decahedrons with an edge size of $40 \pm 5 \mathrm{~nm}$ were used as seeds, thin nanorods with various aspect ratios were obtained. Figure $1 \mathrm{~d}$ shows a gold nanorod with an aspect ratio of 1.8 (length: $112 \mathrm{~nm}$, diameter: $60 \mathrm{~nm}$ ). The selected area electron diffraction (SAED) pattern includes two sets of spots along the zone axes of [001] and [1-1-2] with the growth direction of [110]. It indicates that the rod structure is basically elongated decahedral. The formation mechanism of the rods relies on silver UPD. Because of the three dimensional structure of decahedrons, the tips with the $\{111\}$ facets continuously grow along the [110] direction, and the walls with the $\{100\}$ facets are blocked by selective silver UPD. The silver UPD on the gold $\{100\}$ faces was proven by electrochemical $\mathrm{Cu}$ and $\mathrm{Ag}$ UPD experiments on the rods.
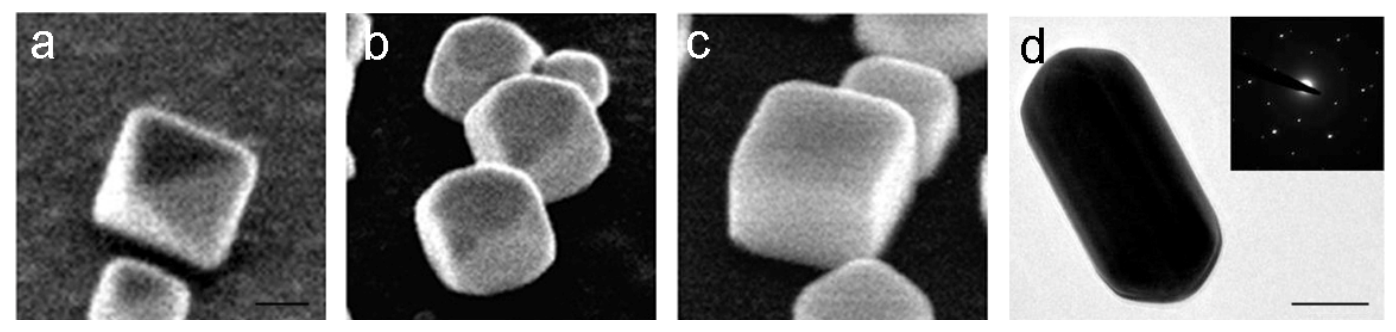

Figure 1. Scanning electron microscopy (SEM) images of gold (a) octahedrons, (b) cuboctahedrons, and (c) cubes. (d) Transmission electron microscopy (TEM) image and SAED pattern of a single gold nanorod. The bars represent $50 \mathrm{~nm}$.

\section{Silver-gold-silver heterometallic nanorods and wires by heteroepitaxial growth}

Epitaxial growth of silver on the gold surface could couple with seed-mediated growth in nanoscale objects [5,9]. Briefly, silver segments were grown on gold decahedral seeds under the present polyol process condition in DEG [5]. Figure 2a shows that each nanorod has a bright strip on the center. The elongated heterometallic nanorods were yielded by using gold rods as seeds. The TEM image of Figure $2 \mathrm{~b}$ shows that the gold and silver segments are clearly separated by contrast difference. The clear SAED pattern and continuous lattice fringe images in high resolution TEM (HRTEM) support that the silver segments are epitaxially grown on the gold surface, due to the small lattice mismatch between silver and gold. Chemical treatment of these heterometallic nanorods with sulfur readily generated $\mathrm{Ag}_{2} \mathrm{~S}-\mathrm{Au}-\mathrm{Ag}_{2} \mathrm{~S}$ semiconductor-metal heterojunctions. 
Under a different polyol process condition in EG, continuous growth of the silver segments from the gold decahedral seeds yielded silver nanowires in an average length of up to 5 $\mu \mathrm{m}$ [6]. The TEM image of a single nanowire shows a projection of the gold decahedron within the straight wire structure (Figure 2c). A clear SAED pattern indicates a pentagonal rod structure corresponding to a superposition of $<112>$ and $<100>$ zone patterns. Continuous lattice fringes were observed throughout the silver and gold regions, indicating epitaxial growth on the gold surface. The silver nanowire diameter was precisely controlled by changing the edge size of the original gold decahedrons. Thin, medium, and thick silver nanowires with average diameters of 63,86 , and $146 \mathrm{~nm}$ were fabricated from the gold octahedrons with edge sizes of 32, 50, and 88 $\mathrm{nm}$, respectively. Such a precise control of nanowire diameters enabled us to study diameter dependency of optical properties. The UV-vis spectrum of the medium nanowires exhibits three distinct extinction peaks at 352,384, and $490 \mathrm{~nm}$ in a visible region, which correspond to bulk, localized, and propagating modes simulated by discrete dipole approximation (DDA) calculations, respectively. In particular, the extinction at $490 \mathrm{~nm}$ originates from the end-on incidence transversal dipole, where the surface plasmon may propagate along the nanowire axis. Figure $2 \mathrm{~d}$ shows a broad side view of the end-on-excitation at $490 \mathrm{~nm}$, illustrating a subwavelength waveguide behavior along the nanowire structure. To the best of our knowledge, this mode is a new transversal dipole that the researchers have not focused on thus far.
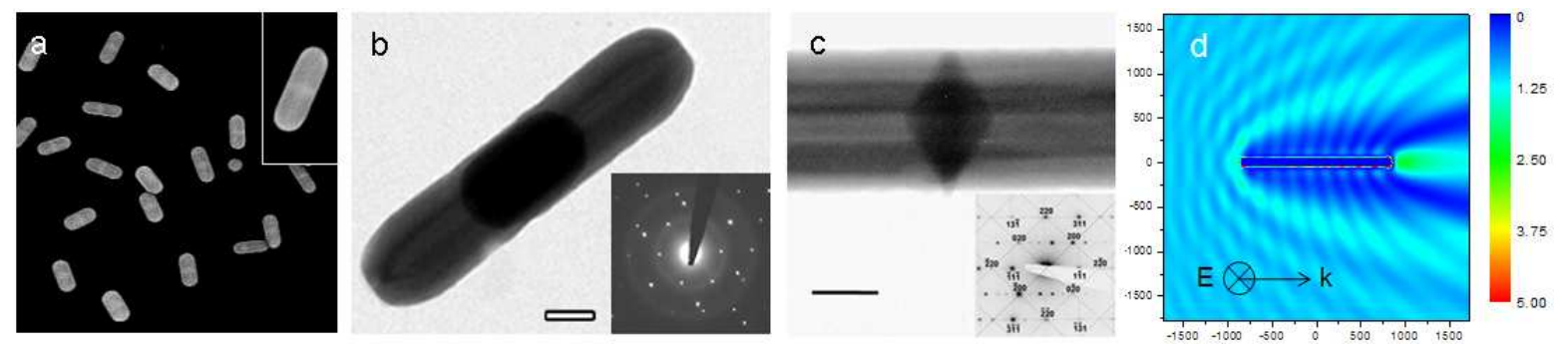

Figure 2. (a) SEM image of Ag-Au-Ag heterometallic nanorods, TEM images and SAED patterns of (b) Ag-Au-Ag elongated nanorods and (c) nanowires, and (d) electric field distribution of the medium silver nanowire. The bars represent $50 \mathrm{~nm}$.

\section{Asymmetric single and symmetric double hollow formation by Galvanic replacement}

Galvanic replacement is one of the powerful methods to synthesize metal hollow nanostructures [10]. Silver nanostructures have normally been used as templates because of the low reduction potential of silver, and noble metals like gold, platinum, and palladium were utilized to replace the original nanostructures. However, most of the hollow structures were restricted to be highly symmetrical, such as hollow spheres, polyhedral nanocages, and tubes, because the Galvanic replacement reaction is rather arbitrary. We have successfully demonstrated the formation of asymmetric hollow structure for the first time in our heterometallic nanorod system [7]. Careful control of the reaction kinetics could yield an asymmetric single hollow as well as a symmetric double hollow in the silver-gold-silver nanorod structure. Addition of a small amount of the gold precursor solution into the rod dispersion randomly generated a single pit on one end of the silver domain in the nanorods. As the addition amount of the gold precursor solution increased, a vacancy was continuously grown from the pit and approached to the full area of one end of each nanorod. Figure 3a shows that the sides of 
each nanorod are different with each other in contrast. The TEM image of a single rod shows that one end of the nanorod is completely empty, and the metallic thin shell covers this vacancy (Figure 3b). The line profile analysis of energy dispersive X-ray spectroscopy (EDX) along the long rod axis confirms asymmetric nature of the hollow position. Further increase of the gold precursor concentration led to the formation of a double hollow structure (Figure 3c). Figure 3d represents that the silver domains are disappeared, and the metal shells surround the original nanorod structure. The gold segment at the center is ellipsoidal because of residual silver layers on the surface.

It is known that gold nanocages intensively scatter light in the near infrared range. The asymmetric single hollow rods have a unique extinction peak centered at $960 \mathrm{~nm}$, in good agreement with those of gold hollow structures over a range of 800-1000 nm. The double hollow rods exhibit two distinct peaks at 830 and $1250 \mathrm{~nm}$ that result from the hollow structure. In both cases, the silver transverse mode is not observed, reflecting complete displacement of the pure silver domains.
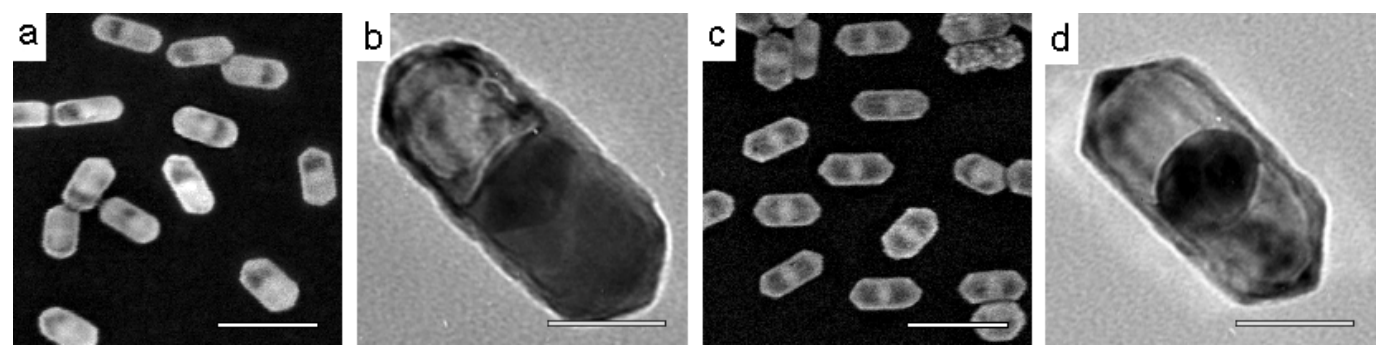

Figure 3. SEM and TEM images of (a,b) asymmetric single hollow rods and (c,d) symmetric double hollow rods. The bars represent $(\mathrm{a}, \mathrm{c}) 200 \mathrm{~nm}$ and $(\mathrm{b}, \mathrm{d}) 50 \mathrm{~nm}$.

\section{CONCLUSION}

Similarity and discrepancy of physical properties in silver and gold make plentiful chemistry and structures in nanoscale objects. Selective underpotential deposition of silver onto the gold $\{100\}$ surface guided the growth direction of nanostructures. Gold polyhedrons with the shapes of octahedral, cuboctahedral, and cubic were synthesized by adjusting the amount of $\mathrm{AgNO}_{3}$. By a combination with seed-mediated growth, gold nanorods with various aspect ratios were fabricated. Epitaxial growth of silver on the gold surface generated silver-gold-silver heterometallic nanorods and nanowires under polyol synthesis conditions. The diameter of silver nanowires could precisely be controlled by using different-sized gold decahedral seeds. Three distinct extinction peaks appeared in the UV-vis spectrum, where the end-on incidence propagating mode was carefully analyzed. Partial Galvanic replacement of silver for gold formed an asymmetric single hollow as well as a symmetric double hollow structure from the silvergold-silver heterometallic nanorods. The asymmetric and symmetric hollows exhibited characteristic optical features in the near infrared range. Such interesting coupling of silver and gold components and the resulting optical properties provide various potential applications such as photonic circuit elements, chemical and biosensors, imaging, drug delivery, and multifunctional catalysis. 


\section{ACKNOWLEDGMENTS}

This work was supported by the Nano R\&D program (2007-02668) and the National

Research Foundation (NRF) grant funded by the Korea Government (MEST) (R11-2007-05000000-0).

\section{REFERENCES}

1. S. J. Hurst, E. K. Payne, L. Qin and C. A. Mirkin, Angew. Chem. Int. Ed. 45, 2672 (2006).

2. S. R. Nicewarner-Peña, R. G. Freeman, B. D. Reiss, L. He, D. J. Peña, I. D. Walton, R. Cromer, C. D. Keating and M. J. Natan, Science 294, 137 (2001).

3. S. E. Habas, H. Lee, V. Radmilovic, G. A. Somorjai and P. Yang, Nat. Mater. 6, 692 (2007).

4. D. Seo, J. C. Park and H. Song, J. Am. Chem. Soc. 128, 14863 (2006).

5. D. Seo, C. I. Yoo, J. Jung and H. Song, J. Am. Chem. Soc. 130, 2940 (2008).

6. J. Jung, D. Seo, G. Park, S. Ryu and H. Song, Angew. Chem. Int. Ed. submitted (2010).

7. D. Seo and H. Song, J. Am. Chem. Soc. 131, 18210 (2009).

8. D. Seo, J. H. Park, J. Jung, S. M. Park, S. Ryu, J. Kwak and H. Song, J. Phys. Chem. C 113, 3449 (2009).

9. D. Seo, C. I. Yoo, J. C. Park, S. M. Park, S. Ryu and H. Song, Angew. Chem. Int. Ed. 47, 763 (2008).

10. Y. Sun, B. Mayers and Y. Xia, Adv. Mater. 15, 641 (2003). 